\title{
MAXIMAL CANCELLATIVE SUBSEMIGROUPS AND CANCELLATIVE CONGRUENCES
}

\author{
MOHAN S. PUTCHA
}

\begin{abstract}
A subsemigroup $T$ of a commutative semigroup $S$ is called a mild ideal if for any $a \in S, a T \cap T \neq \varnothing$. It is shown here that any maximal cancellative subsemigroup $T$ of a commutative, idempotentfree, archimedean semigroup $S$ must be a mild ideal of $S$. Maximal cancellative subsemigroups exist in abundance due to Zorn's lemma. It is also shown that if $T$ is mild ideal of a commutative semigroup $S$, then every cancellative congruence of $T$ has a unique extension to a cancellative congruence of $S$.
\end{abstract}

1. Maximal cancellative subsemigroups. Let $S$ be a commutative archimedean semigroup with no idempotents. Let $A$ be a cancellative subsemigroup of $S$. By the Hausdorff maximal principle (Zorn's lemma), there will exist a maximal ${ }^{1}$ cancellative subsemigroup $T$ such that $A \subseteq T$. In particular if $a \in S$, then the cyclic semigroup $\langle a\rangle$ is cancellative, and hence there exists a maximal cancellative subsemigroup of $S$ containing $a$. In what follows, $Z^{+}$denotes the set of positive integers.

We start with

Lemma 1.1. Let $S$ be a commutative, archimedean, idempotent-free semigroup and let $T$ be a maximal cancellative subsemigroup of $S$. Then for any $a \in S \backslash T$, there exists $i \in Z^{+}$and $t_{1}, t_{2} \in T^{1}, u \in T$, such that $a^{i} t_{1} u=t_{2} u$ but $a^{i} t_{1} \neq t_{2}$.

Proof. We use, without further comment, a result of Tamura (see [2] or [3]) that for any $a, b \in S, a b \neq b$. Now let $a \in S \backslash T$. By maximality of $T$, the semigroup generated by $a$ and $T$ is not cancellative. So there exist nonnegative integers $j, k$ and $t_{1}, t_{2} \in T^{l}, x \in S$, such that $a^{j} t_{1} \neq$ $a^{k} t_{2} ; a^{j} t_{1} x=a^{k} t_{2} x$. If $j=k$, then $t_{1} a^{j} x=t_{2} a^{j} x$. Since $S$ is archimedean,

Received by the editors November 16, 1973.

AMS (MOS) subject classifications (1970). Primary $20 \mathrm{M} 10$.

Key words and phrases. Semigroups, commutative, archimedean, maximal cancellative congruence, mild ideal.

1 Maximal as a cancellative subsemigroup, not as a subsemigroup. 
$t_{1} y=t_{2} y$ for some $y \in T$ whence $t_{1}=t_{2}$. So $a^{j} t_{1}=a^{k} t_{2}$, a contradiction. So $j \neq k$. Let us say $j>k$. Then $i=j-k \in Z^{+}$. Now $a^{i} t_{1} \neq t_{2}$ lest $a^{j} t_{1}=$ $a^{k} t_{2}$. Now $a^{i} t_{1}\left(a^{k} x\right)=t_{2}\left(a^{k} x\right)$. Since $S$ is archimedean, $a^{i} t_{1} u=t_{2} u$ for some $u \in T$. This proves the lemma.

Definition. Let $S$ be a commutative semigroup and $T$ a subsemigroup of $S$. Then $T$ is a mild ideal of $S$ if for every $a \in S, a T \cap T \neq \varnothing$.

Theorem 1.2. Let $S$ be a commutative, archimedean, idempotent-free semigroup and $T$ a maximal cancellative subsemigroup of $S$. Then $T$ is a mild ideal of $S$.

Proof. Let $a \in S \backslash T$. We must show that $a T \cap T \neq \varnothing$. By Lemma 1.1, there exists $i \in Z^{+}$such that $a^{i} T \cap T \neq \varnothing$. Choose $i$ minimal. We assume $i>1$ and obtain a contradiction. Now $a^{i} t \in T$ for some $t \in T$. Let $b=a t$. Then $b \notin T$ but $b^{i} \in T$. Again by Lemma 1.1 there exist $j \in Z^{+}$and $t_{1}, t_{2}$ $\in T^{1}, u \in T$, such that $b^{j} t_{1} u=t_{2} u$ but $b^{j} t_{1} \neq t_{2}$. So $b^{j} \notin T$. Thus $i+j$. So there exist $k \in Z^{+}$and an integer $l(l \geq 0)$ such that $j=i l+k, k<i$. Let $c=b^{i l}$. Then $c \in T^{1}$. Also $b^{k}\left(c t_{1} u\right)=b^{j} t_{1} u=t_{2} u \in T$. Therefore $b^{k} T \cap$ $T \neq \varnothing$. Since $b \in a T, a^{k} T \cap T \neq \varnothing$. But this contradicts the minimality of $i$. So it must be that $T$ is a mild ideal of $S$.

Remark. In general there is no hope of $T$ being an ideal of $S$. In fact Professor Takayuki Tamura has shown that there exist commutative archimedean semigroups with no cancellative ideals. He has also obtained necessary and sufficient conditions for the existence of a cancellative ideal.

A commutative, cancellative, idempotent-free archimedean semigroup is known as an n-semigroup. They have been well studied by Tamura and others. Let $T$ be a commutative, cancellative idempotent-free semigroup. Suppose there is a partial order $\leq$ defined on $T$ such that $(T, \leq)$ is a partially ordered semigroup, $\leq$ is positive ( $a b \geq a, b$ for all $a, b \in T$ ), and $T$ is $\leq-$ strongly archimedean, i.e. $a<b$ implies that for any $c \in T$ there exists $i \in Z^{+}$such that $a^{i} c<b^{i}$. Then according to the author [1], one can in a very natural way embed $T$ in an $n$-semigroup $N(T, \leq)$ called the quotient $n$-semigroup of $T$. In particular, if $S$ is a commutative, archimedean, idempotent-free semigroup and $T$ is a cancellative subsemigroup, then we can consider $\leq$ on $T$ induced by division in $S$. Then $(T, \leq)$ has all the properties discussed above. So we can construct the quotient $n$-semigroup $N(T, \leq)$ containing $T$. Precisely, if $G$ is the quotient group of $T$, then $N(T, \leq)=$ $\left\{x \mid x \in G, x=a b^{-1}\right.$ for some $a, b \in T$ and $\left.b<a\right\}$.

On the other hand, if $\sigma$ is the finest cancellative congruence on $S$ (i.e. 
$a \sigma b$ iff $a c=b c$ for some $c \in S$ ), then $S^{\prime}=S / \sigma$ is an $n$-semigroup [3]. The relationship between these two ways of associating $n$-semigroups with $S$ lies in the following result.

Theorem 1.3. Let $S$ be a commutative, archimedean, idempotent-free semigroup and let $T$ be a maximal cancellative subsemigroup of $S$. If $S^{\prime}$ is the greatest cancellative image of $S$, then $S^{\prime} \cong N(T, \leq)$ where $\leq$ is the partial order on $T$ induced by division in $S$.

Proof. Let $\phi: S \rightarrow S^{\prime}$ be the natural homomorphism. Let $a, b \in T$ and $\phi(a)=\phi(b)$. Then $a c=b c$ for some $c \in S$. Since $S$ is archimedean, $a u=b u$ for some $u \in T$ and consequently $a=b$. So $T \cong \phi(T)=T^{\prime}$. Let $G$ be the quotient group of $S^{\prime}$ and $H$ the quotient group of $T^{\prime}$. Then $N(T, \leq) \cong N\left(T^{\prime}, \leq^{\prime}\right) \subseteq H \subseteq G$. Here $\leq^{\prime}$ is induced by $\leq$ on $T$. First let $a$, $b \in T, b<a$. Then $b x=a$ for some $x \in S$. Hence $\phi(a) \phi(b)^{-1}=\phi(x) \in S^{\prime}$. So $N\left(T^{\prime}, \leq^{\prime}\right) \subseteq S^{\prime}$. Conversely let $x \in S$. By Theorem 1.2, $T$ is a mild ideal of $S$. Therefore there exist $a, b \in T$ such that $b x=a$. Then $b<a$ and $a b^{-1} \in N(T, \leq)$. Now $\phi(x)=\phi(a) \phi(b)^{-1} \in N\left(T^{\prime}, \leq^{\prime}\right)$. So $N\left(T^{\prime}, \leq^{\prime}\right)=$ $S^{\prime}$, proving the theorem.

Remark. $T=N(T, \leq)$ iff $\leq$ is equal to division in $T$.

Problem. Let $S$ be a commutative, archimedean, idempotent-free semigroup and $T$ a maximal cancellative subsemigroup of $S$. Is $T$ necessarily an $n$-semigroup?

2. Cancellative congruences. Mild ideals are also nice when dealing with cancellative congruences (i.e. congruences $\sigma$ on $S$ such that $S / \sigma$ is cancellative).

Theorem 2.1. Let $S$ be a commutative semigroup and $T$ a mild ideal of $S$. Then every cancellative congruence on $T$ extends uniquely to a cancellative congruence on $S$. Thus there is a one-to-one correspondence between cancellative congruences of $S$ and those of $T$.

Proof. Let $\sigma$ be a cancellative congruence on $T$. Define $\hat{\sigma}$ on $S$ as follows: for $a, b \in S, a \hat{\sigma} \cdot b$ iff $a t \sigma b t$ for some $t \in T$ such that $a t, b t \in T$. Evidently $\hat{\sigma}$ is symmetric. It is reflexive since $T$ is a mild ideal. Let $a$, $b, c \in S$ such that $a \hat{\sigma} b \hat{\sigma} c$. Then for some $t_{1}, t_{2} \in T$ and $a t_{1}, b t_{1}, b t_{2}$, $c t_{2} \in T, a t_{1} \sigma \cdot b t_{1}$ and $b t_{2} \sigma c t_{2}$. With $t=t_{1} t_{2}, a t \sigma c t$ and $a t, c t \in T$. So $\hat{\sigma}$ is an equivalence relation on $S$. Since $\sigma$ is cancellative, $\hat{\sigma} \mid T=\sigma$. Next let $a, b \in S, a \hat{\sigma} b$. Then $a t_{1} \sigma b t_{1}$ for some $t_{1} \in T$ such that $a t_{1}$, $b t_{1} \in T$. Let $c \in S$. Then $c t_{2} \in T$ for some $t_{2} \in T$. Thus $a t_{1} c t_{2} \sigma \cdot b t_{1} c t_{2}$, 
and so $a c\left(t_{1} t_{2}\right) \sigma \cdot b c\left(t_{1} t_{2}\right)$ showing $a c \hat{\sigma} \cdot b c$. So $\hat{\sigma}$ is a congruence on $S$. Finally let $a, b, c \in S, a c \hat{\sigma} b c$. So $a c t_{1} \sigma b c t_{1}$ for some $t_{1} \in T$ such that $a c t_{1}$ and $b c t_{1} \in T$. Now $c t_{2} \in T$ for some $t_{2} \in T$. Thus $a\left(c t_{2} t_{1}\right)$ $\sigma \cdot b\left(c t_{2} t_{1}\right)$ whence $a \hat{\sigma} b$. So $\hat{\sigma}$ is a cancellative congruence on $S$.

Next let $\sigma_{1}, \sigma_{2}$ be two cancellative congruences on $S$ such that $\sigma_{i} \mid T$ $=\sigma \cdot(i=1,2)$. Let $a, b \in S, a \sigma_{1} b$. Then for some $t_{1}, t_{2} \in T, a t_{1}, b t_{2} \in T$. So $a t, b t \in T$ with $t=t_{1} t_{2}$. Moreover, at $\sigma_{1} b t$, whence at $\sigma$ bt. Now it must be that at $\sigma_{2}$ bt whereupon a $\sigma_{2} b$. So $\sigma_{1} \subseteq \sigma_{2}$. Similarly $\sigma_{2} \subseteq \sigma_{1}$ whence $\sigma_{1}=\sigma_{2}$. Conversely, any cancellative congruence on $S$ has a restriction to a cancellative congruence on $T$.

Remark. Cancellative congruences of $n$-semigroups have been determined in different ways by Tamura [4]. Of course the cancellative congruences of $S$ are derived from those of $S^{\prime}$. But Theorem 2.1 tells how they can also be derived from those of any maximal cancellative subsemigroup $T$ of $S$. Note that $T$ is a mild ideal of $N(T, \leq)$. Therefore the cancellative congruences of $T$ are the restrictions of the cancellative congruences of the $n$-semigroup $N(T, \leq)$.

\section{REFERENCES}

1. M. S. Putcha, Positive quasi-orders on semigroups, Duke Math. J. 40(1973), 857-869.

2. T. Tamura, Commutative nonpotent archimedean semigroup with cancellation law. I, J. Gakugei Tokushima Univ. 8 (1957), 5-11. MR 20 \#3224.

3. - Construction of trees and commutative archimedean semigroups, Math. Nachr. 36 (1968), 257-287. MR 37 \#6222.

4. - $\longrightarrow$-congruences of $\pi$-semigroups, J. Algebra 27 (1973), 11-30.

DEPARTMENT OF MATHEMATICS, UNIVERSITY OF CALIFORNIA, BERKELEY, CALIFORNIA 94720 